\title{
Was macht die regionale Praxis?
}

\author{
Die derzeitige Diskussion von Land- und Ernährungswirtschaft in Verbindung \\ mit nachhaltiger Entwicklung ist durch einen Spagat charakterisiert: Der Diskus- \\ sion um die Globalisierung von (Agrar-)Märkten, politische Rahmenbedingungen \\ und Produktionsphilosophien steht dabei eine Vielzahl von praktischen Ansät- \\ zen und Konzepten auf lokaler und regionaler Raumebene gegenüber. Was \\ bleibt jenseits der Individualität dieser Nachhaltigkeitsversuche?
}

$\mathrm{V}$ Von Reimar Molitor iele Projekte treiben pragmatisch die Umsetzung einer nachhaltigen Land- und Ernährungswirtschaft vor Ort voran. Als Basis der folgenden Analyse dient eine Datenbank von 417 Projekten, die vom IÖW im Rahmen der Studie „Regionale Good Practices einer nachhaltigen Raum- und Siedlungsentwicklung" angelegt wurde. Rund 70 davon weisen einen unmittelbaren Bezug zu Landwirtschaft und Ernährung auf (1). Eine Betrachtung dieser individuell gestalteten Projekte auf regionaler Ebene wirft die Frage nach ihrer Vergleichbarkeit hinsichtlich räumlicher Kontextbedingungen, Akteursbezügen, Instrumenten etc. auf. Die Individualität der einzelnen projektbezogenen „Zutaten“ scheint dabei auf den ersten Blick eine strategische Operationalisierung auszuschließen.

Die Untersuchung regionaler Projekte einer nachhaltigen Entwicklung im Bereich Landwirtschaft und Ernährung konzentrierte sich daher in den vergangenen Jahren stark auf die Begleitung der Projekte unter verschiedenen Leitmotiven wie etwa Kooperation und Partizipation (vgl. den Beitrag von Witzel/Tischer in diesem Heft). Zeitgleich konzentrierte sich die politische Flankierung dieser Projekte auf die Förderung und Aufbereitung sogenannter ,good“ bzw. „,best practices“. Während der zuerst genannte Komplex transferierbare Einzelaspekte einer nachhaltigen Regionalentwicklung identifizierte, ermöglichte der zweite Komplex vor allem den inter- und intraregionalen Austausch über Inhalte und Organisationsformen der Projekte und beförderte dadurch in Deutschland eine regionale Imitationsdynamik.

\section{- Nachhaltige Entwicklung als kleinster gemeinsamer Nenner}

Durchgängige Gemeinsamkeit der Projekte ist dabei das Bekenntnis zum Leitbild Nachhaltige
Entwicklung. Das Verständnis und die Auslegung des Leitbildes variiert jedoch stark: Die Praxis einer nachhaltigen Land- und Ernährungswirtschaft auf regionaler Ebene ist gekennzeichnet durch eine enorme Vielfalt bezüglich ihrer inhaltlichen Programmatik und organisatorischen Struktur. Die Bemühungen vor Ort reichen vom professionellen sektorübergreifenden Management regionaler Entwicklungsprozesse bis hin zum einzelbetrieblichen Produktmarketing. Jenseits des kleinsten gemeinsamen Nenners lassen sich jedoch weitere Gemeinsamkeiten identifizieren, die Erkenntnisse für eine strategische Operationalisierung einer nachhaltigen Land- und Ernährungswirtschaft auf regionaler Ebene liefern.

\section{Thematische Raumzuschnitte}

In direkter Abhängigkeit von der thematischen Schwerpunktwahl der Projekte stehen die räumlichen Zuschnitte der Projekt-Regionen. Gemäß dem Integrationsanspruch der Zieldimensionen Ökologie, Ökonomie und Soziales einer nachhaltigen Entwicklung kommt es innerhalb der Projekte zu einer flexiblen räumlichen Verknüpfung von

- funktionalen Raumdimensionen (zum Beispiel wirtschaftliche Austauschbeziehungen),

- stofflichen Raumdimensionen (Stoffkreisläufe und Energienutzung entlang von landwirtschaftlichen Produktlinien) als auch

- natur- und kulturräumlichen Dimensionen (Qualitäts- und Herkunftssicherung, Landschaftspflege).

Auffälligstes Merkmal ist dabei der Versuch der Rückkopplung der Stufen Produktion, Verarbeitung und (mit Einschränkung) Handel an regionale Raumbezüge. Es scheint hier der Schluss zulässig, dass die praktische Interpretation einer nachhaltigen Land- und Ernährungswirtschaft transsektorale Regionalisierungsstrategien favo- risiert (2). Ergänzend ist festzuhalten, dass auch die ökologischen Nutzen unter der Überschrift Regionalisierung vermarktet werden, so zum Beispiel die Verringerung des Transportaufwands und die daraus resultierende Kohlendioxid-Reduzierung im Rahmen der Produktprofilierungsstrategie „Aus der Region - für die Region“ oder landschaftsökologische Effekte im Rahmen der landwirtschaftlichen Pflege der überwiegend regional definierten Kulturlandschaften.

\section{- Neukonstellation der Akteure}

Ein weiteres Phänomen stellt die veränderte Konstellation von Akteuren dar. In enger Abhängigkeit von den themenspezifischen Raumzuschnitten ist entlang des jeweiligen Projektthemas eine neue Vernetzung von regionalen Akteuren zu beobachten (2). Merkmal dieser Neukonstellation von Akteuren ist die Einbindung von individuellen und kollektiven Akteuren aus regionalen Anrainerbereichen der Landwirtschaft. Bevorzugt sind dies Naturschutzverbände, kirchliche Gruppierungen, Verbraucherverbände und touristisch orientierte Verbände. Sie lösen sowohl durch projektinterne als auch regionsbezogene Kommunikationsprozesse die reduktionistische Wahrnehmung der Landwirtschaft als einzelbetriebliche Produktionseinheit auf. An ihre Stelle tritt eine integrierte Sichtweise der regionalen Interdependenzen etwa zwischen landwirtschaftlicher Produktion, Naherholung und Tourismus, Versorgung, Landschaftspflege und Naturschutz. Häufig gewählte organisatorische Formen der Einbindung dieser Anrainerbereiche sind Vereine, Projektbeiräte oder regionale Entwicklungsgesellschaften. Nicht selten etablieren sich dabei in den Regionen Macht- und Entscheidungsstrukturen, die jenseits der bereits existierenden Interessenvertretungen und Planungsträger wie zum Beispiel Landwirtschaftskammern arbeiten. Dabei ist häufig der schleichende Aufbau von Konkurrenzstrukturen zu beobachten, die den Prozess der langfristigen Akzeptanz und Verstetigung einer nachhaltigen Land- und Ernährungswirtschaft zumindest gefährden.

\section{- Nachhaltige Zweckbündnisse?}

Ein ambivalentes Merkmal ist der Bereich des landwirtschaftlichen Produktmarketings. Viele Projekte nutzen sowohl die erweiterte Raumdefinition als auch die Einbeziehung neuer regionaler Akteure als Legitimationsbasis einer nachbaltigen Land- und Ernährungswirtschaft. Dabei gewährleistet in Einzelfällen schon die 


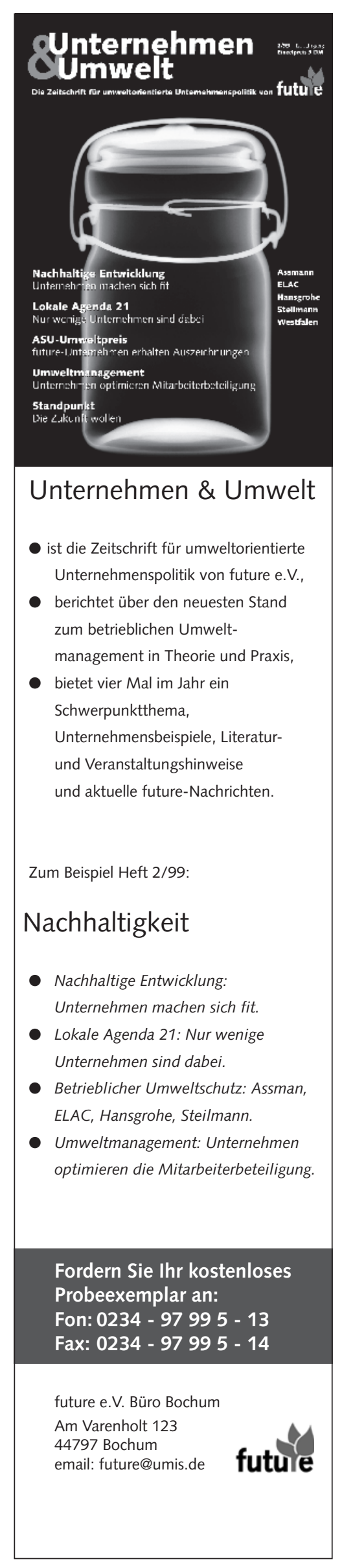

formale Projektpartizipation regionaler Akteure aus dem Naturschutz- und Umweltbereich eine Ausweisung mit dem Prädikat nachhaltig. Dieser Sachverhalt unterläuft vor allem Ansätze jener Initiativen, die über Jahre hinweg mit hohem Kommunikations- und Abstimmungsaufwand im regionalen Nachhaltigkeitsdiskurs eine inhaltliche und organisatorische Kongruenz zwischen Erzeugungs-, Verarbeitungs- und Vermarktungskriterien erreicht haben. In einigen Projekten ist darüber hinaus eine Doppelfunktion des landwirtschaftlichen Produktmarketings gegeben: Neben der unmittelbaren „nachhaltigen“ Produkt-Differenzierungsstrategie fungiert die Etablierung von regionalen Dachmarken landwirtschaftlicher Erzeugnisse oftmals als integraler Bestandteil eines umfassenden Regionenmarketings (Brucker Land, MüblenGarten, Rhöner Charme usw.). Inwiefern hierbei inhaltliche Dimensionen einer nachhaltigen Land- und Ernährungswirtschaft berücksichtigt sind, lässt sich nur für den jeweiligen Einzelfall anhand der spezifischen Entwicklungskonzeptionen und Kriterienkataloge nachvollziehen.

\section{Immer nur ländlicher Raum?}

Zum Abschluss soll noch auf das besondere Merkmal des Regionenverständnisses im Zusammenhang mit einer nachhaltigen Land- und Ernährungswirtschaft eingegangen werden. Die untersuchten Projekte weisen einen eindeutigen Schwerpunkt im Bereich der ländlich strukturierten Regionen auf. Nur wenige Initiativen haben einen direkten Bezug zum Verdichtungsraum. Diese Feststellung wirkt auf den ersten Blick trivial, geht es doch in der bisherigen Diskussion um Schlagwörter wie regionale Kulturlandschaft, regionale Wirtschaftskreisläufe oder Erhalt der Biotopund Artenvielfalt - nur: muss dies zwangsläufig und nahezu ausschließlich im ländlich strukturierten Raum ablaufen? Stark vernachlässigt werden in diesem Zusammenhang die Potenziale einer nachhaltigen Land- und Ernährungswirtschaft, die (noch) im Stadt-Umland-Bereich der Verdichtungsräume brachliegen (vergleiche den Beitrag von Fiebig in diesem Heft). Besonders im Rahmen von Lokale Agenda 21-Prozessen haben in jüngster Vergangenheit Städte wie Frankfurt, Köln oder Wuppertal die Land- und Ernährungswirtschaft als Thema einer nachhaltigen (Stadt-!)Entwicklung wieder entdeckt. Hier wird das oben ausgeführte inhaltliche Verständnis um Dimensionen wie Flächendruck oder Konflikte mit Naberholungssuchenden erweitert. Gerade in diesem Spannungsfeld stellt die Nähe zum Konsumenten einen entscheidenden Prüfstein für die dauerhafte Etablierung einer regional orientierten nachhaltigen Land- und Ernährungswirtschaft dar: Gelingt es, das Verhältnis von Stadt und Umland in Bezug auf eine nachhaltige Land- und Ernährungswirtschaft, zum Beispiel durch regionale Angebotsbündelungen, Professionalisierung der Vermarktung, Erhalt von mittelständischen Verarbeitungsstrukturen, Erschließung des landwirtschaftlichen Dienstleistungsbereichs oder Optimierung der Landschaftspflege strategisch zu operationalisieren? Die Frage kann an dieser Stelle nicht beantwortet werden. Nur eins scheint sicher: Gelingt diese Etablierung auch auf lange Sicht nicht, bleiben zwangsläufig auch die oben angeführten Projekte in den eher ländlich strukturierten Regionen auf dem Status von Leuchtturmprojekten stehen. Es bleibt daher kritisch zu beobachten, wie ernst es Politik und Verbraucher mit einer nachhaltigen Entwicklung der Land- und Ernährungswirtschaft in der Praxis halten.

\section{Anmerkungen}

(1) Vgl. Bundesamt für Bauwesen und Raumordnung (Hrsg.): Handbuch Regionale Good Practices einer nachhaltigen Raum- und Siedlungsentwicklung. Werkstatt: Praxis Nr. 1/2000; Kurzfassung unter: www.zukunftsregionen.de (2) Vgl. Nischwitz, G./ Molitor, R.: Regionale Nachfragepotentiale in der Produktlinie Ernährung. Schriftenreihe des IÖW Nr.135/98, Berlin 1998; Molitor, R.: Nachhaltige Regionalentwicklung. Von der regulativen Idee zur Umsetzung vor Ort. Schriftenreihe des IÖW Nr.146/00, Berlin 2000 (im Erscheinen).

\section{Der Autor}

Dr. Reimar Molitor ist wissenschaftlicher Mitarbeiter im Forschungsfeld Regionale Wirtschaftspolitik/ Nachhaltige Regionalentwicklung im IÖW. Kontakt: IÖW Regionalbüro Nordrhein-Westfalen, Völklinger Str. 9, 42285 Wuppertal, Tel. 0202/ 80530, Fax 0202/ 83402,

E-mail: reimar.molitor@wuppertal.ioew.de 
(c) 20I0 Authors; licensee IÖW and oekom verlag. This is an article distributed under the terms of the Creative Commons Attribution Non-Commercial No Derivates License (http://creativecommons.org/licenses/by-nc-nd/3.o/), which permits unrestricted use, distribution, and reproduction in any medium, provided the original work is properly cited. 